\title{
Comparison between Autogenous Bone Graft and Allogenous Cancellous Bone Graft in Medial Open Wedge High Tibial Osteotomy with 2-Year Follow-up
}

Sung Won Cho, MD, Dong Hwi Kim, MD, Gwang Chul Lee, MD, Sang Hong Lee, MD, and Sang Ha Park, MD Department of Orthopaedic Surgery, Chosun University Hospital, Gwangju, Korea

\begin{abstract}
Purpose: To compare the radiographic and clinical results of medial open wedge high tibial osteotomy (OWHTO) using autogenous bone graft and allogenous cancellous bone graft for medial compartment osteoarthritis of the knee with two-year follow-up.

Materials and Methods: Fifty-one patients (52 knees) who underwent medial OWHTO from October 2007 to April. 2010 were included in the study. The patients were divided into group I $(n=29)$ that received an autogenous tricortical bone graft and group II ( $\mathrm{n}=23)$ that received an allogenous cancellous bone chip graft. The radiographic parameters (preoperative anatomical and mechanical femorotibial angles, modified tibial bone varus angle, and posterior tibial slope), clinical parameters, bone union period, and complications were evaluated from medical records.

Results: The radiographic and clinical outcomes did not show significant difference between two groups. The average bone union period was 11.7 weeks in group I and 12.1 weeks in group II. The visual analog scale score on the first postoperative day was significantly higher in group I than group II.

Conclusions: Medial OWHTO using allogenous cancellous bone graft for medial compartment osteoarthritis of the knee can be considered as an alternative treatment method that provides equivalent radiographic and clinical results of OWHTO using autogenous bone graft and causes less immediate postoperative pain.
\end{abstract}

Keywords: Knee, Medial compartment, Osteoarthritis, High tibial osteotomy, Bone graft

\section{Introduction}

High tibial osteotomy has been recognized as a standard procedure for the treatment of medial compartment osteoarthritis of the knee with varus deformity. Although it can be performed with either an open or closed wedge technique, open wedge high tibial osteotomy (OWHTO) is being employed with increasing frequency because it allows for easier and more accurate de-

Received January 8, 2013; Revised (1st) March 20, 2013;

(2nd) June 25, 2013; (3rd) July 13, 2013; Accepted July 17, 2013

Correspondence to: Dong Hwi Kim, MD

Department of Orthopaedic Surgery, Chosun University Hospital, 365

Pilmun-daero, Dong-gu, Gwangju 501-717, Korea

Tel: +82-62-220-3147, Fax: +82-62-226-3379

E-mail: oskdh@chosun.ac.kr

This is an Open Access article distributed under the terms of the Creative Commons Attribution Non-Commercial License (http://creativecommons.org/licenses/by-nc/3.0/) which permits unrestricted non-commercial use, distribution, and reproduction in any medium, provided the original work is properly cited. formity correction. However, OWHTO requires bone grafting to fill the bone defect created by osteotomy. Autogenous bone grafts harvested from the iliac crest have been considered as the standard graft material, but prolonged operating time and severe donor site pain are the primary drawbacks ${ }^{1)}$. To overcome these disadvantages, a variety of solutions have been suggested in many studies: allogenous bone grafting; leaving the osteotomy opening unfilled; and using synthetic materials such as tricalcium phosphate $^{2)}$, bicalcium phosphate granule, acrylic cement ${ }^{3)}$, and hydroxyapatite $^{4)}$. Still, there are few comparison studies of the allogenous grafts and autogenous grafts ${ }^{5}$. In this study, we compared the radiographic and clinical outcomes of medial OWHTO using an allogenous cancellous bone graft with that using an autogenous bone graft for two years of follow-up period. Our hypothesis was that autogenous bone grafts would yield more satisfying radiolographic and clinical results. 


\section{Cho et al. Auto and Allogenous Cancellous Bone Graft in HTO}

\section{Materials and Methods}

\section{Patient Selection and Grouping}

Between October 2007 and April 2010, medial OWHTO was performed in patients with the following conditions: $\geq$ KellgrenLawrence grade II osteoarthritis in the medial compartment and varus mechanical femorotibial alignment on the preoperative whole-leg plain radiograph; cartilage defect of the medial condyle of the femur/tibia without abnormal findings in the lateral compartment on magnetic resonance imaging; and pain and swelling that lasted for $\geq 3$ months. Of the total patients, 52 knees of 51 patients in whom the Puddu plate (Arthrex, Naples, FL, USA) was used for fixation and the follow-up examination was available for $\geq 2$ years were retrospectively reviewed by two orthopedic surgeons. According to the type of graft used, the patients were divided into two groups, autogenous bone graft group (group I, 29 knees) and allogenous bone graft group (group II, 23 knees). The patients were asked to choose the type of graft after being informed on the advantages and disadvantages of each type.

\section{Gender and Age Distribution}

In group I $(n=29), 7$ knees were from male patients and 22 knees were from female patients with a mean age of 53.9 years (range, 40 to 75 years). In group II $(n=23), 3$ knees were from male patients and 20 knees were from female patients with a mean age of 58.7 years (range, 44 to 69 years). The mean body mass index (BMI) was $26.3 \pm 2.86$ in group I and $25.1 \pm 3.32$ in group II. No significant intergroup difference was found in age and BMI. Autogenous graft was preferred in males than females $(\mathrm{p}=0.042)$. The preoperatively planned correction angle was not significantly different between group I and group II $\left(8.3^{\circ} \pm 2.59^{\circ}\right.$ vs. $\left.7.7^{\circ} \pm 2.78^{\circ} ; \mathrm{p}=0.411\right)$ (Table 1$)$.

\section{Surgical Technique}

Under general or spinal anesthesia, the patient was placed in the supine position on the radiolucent operating table with a tourniquet applied to the injured limb. Preoperatively, anatomical and mechanical femorotibial angles, modified tibial bone varus angle, and posterior tibial slope were assessed. Regarding the correction of the mechanical axis, it was planned to be located at $62 \%$ lateral from medial end of the tibial plateau. ${ }^{6}$ An arthroscopic examination was performed to confirm the preoperative findings including medial compartment arthritis, intraarticular lesions, and intact lateral compartment. An 8-cm longitudinal skin incision was made $1-2 \mathrm{~cm}$ medial to the medial border of the tibial tuberosity and $1 \mathrm{~cm}$ below the joint line. The superficial medial collateral ligament and pes anserinus were detached as a single flap from the proximal tibia. During the procedure, the distal attachment of the superficial medial collateral ligament was completely separated from the bone to reduce pressure on the medial compartment of the knee. With the planned osteotomy site fully exposed, a steinmann pin was inserted from the superomedial portion of the tibial tuberosity where the patellar tendon is detached for avoiding tendon injury during osteotomy toward the tip of fibular head under image intensifier guidance (Fig. 1A) Then, with the knee brought to a figure-of- 4 position, the guiding block was rotated to make the osteotomy line in parallel with the posterior tibial slope on the sagittal plane. Another steinmann pin was inserted and the guiding block was fixated to the medial side of the tibia using a head pin. After completely resecting the medial cortex of the tibia using a reciprocal saw, osteotomy was advanced using an osteotome to $5 \mathrm{~mm}$ medial to the lateral cortex of the tibia (Fig. 1B). With two osteotomes placed at the osteotomy site, another osteotome was inserted in between the two to prevent the osteotomy line from extending to the joint line and lateral cortex of tibia. When the osteotomy site was opened to the preoperatively planned extent by gradually applying a valgus force (Fig. 1C), the medial tibia was fixated with the Puddu plate at a site $1-\mathrm{cm}$ anterior to the posteromedial border of the osteotomy site $^{7)}$ (Fig. 1D). In group I, two tricortical bone grafts

Table 1. Demographic Data of the Patients

\begin{tabular}{lccc}
\hline \multicolumn{1}{c}{ Variable } & Autogenous bone graft group $(\mathrm{n}=29)$ & Allogenous bone graft group $(\mathrm{n}=23)$ & $\mathrm{p}$-value \\
\hline Age $(\mathrm{yr})$ & $53.9 \pm 9.05$ & $58.6 \pm 8.02$ & 0.053 \\
Male & $7(24)$ & $3(13)$ & 0.042 \\
$\quad$ Average age & $54 \pm 8.87$ & $60 \pm 8.12$ & $20(87)$ \\
Female & $22(76)$ & $57 \pm 10.17$ & 0.570 \\
Average age & $53 \pm 10.30$ & $25.12 \pm 3.32$ & 0.171 \\
Body mass index & $26.32 \pm 2.86$ & $7.7 \pm 2.78$ & 0.411 \\
Preoperative correction angle & $8.3 \pm 2.59$ & & \\
\hline
\end{tabular}

Values are presented as mean \pm standard deviation or number (\%). p-value $<0.05$. 


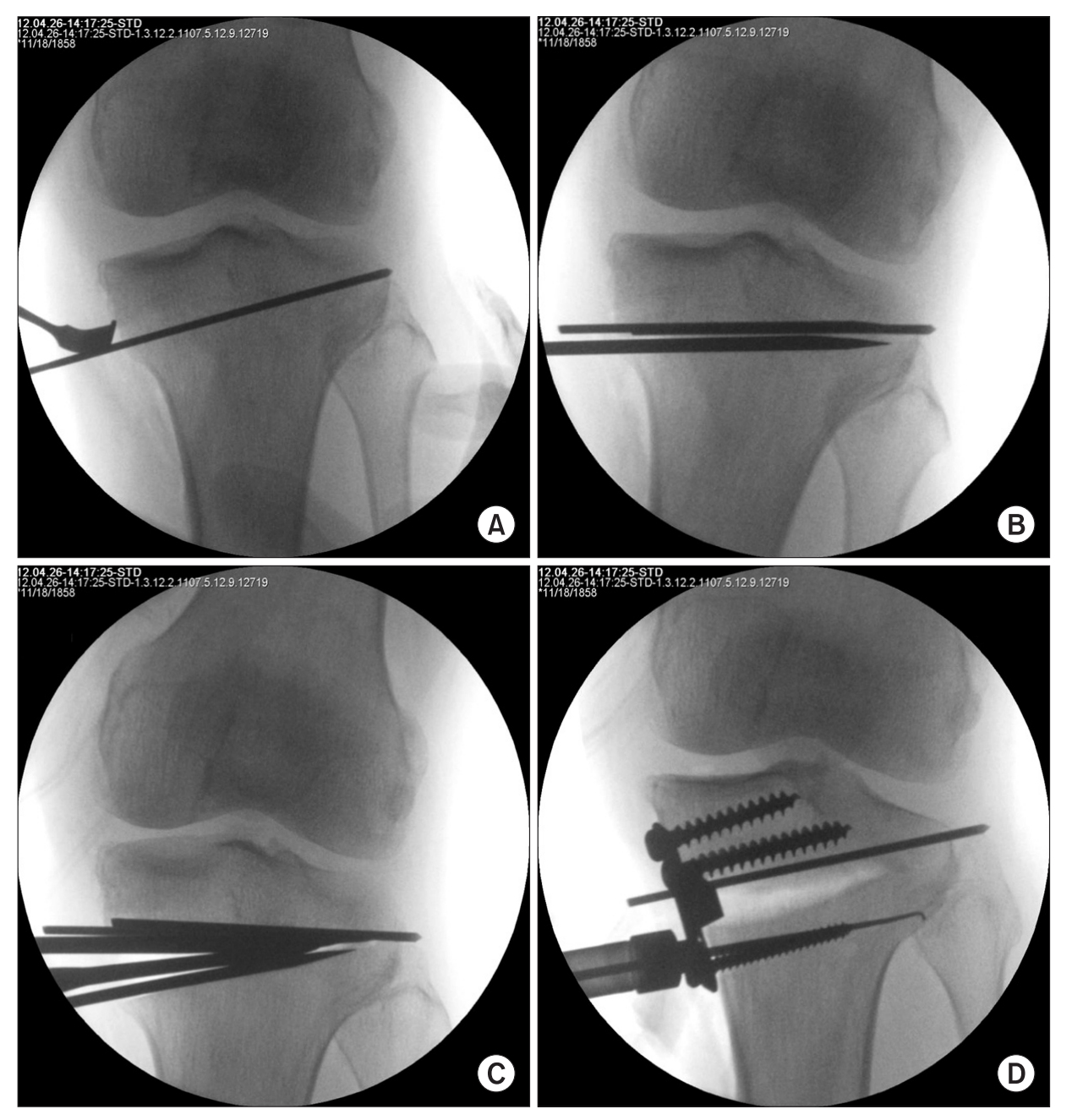

Fig. 1. Surgical technique. (A) A guide pin was placed from the superomedial portion of the tibial tuberosity to the fibular head approximately $1 \mathrm{~cm}$ below the lateral articular margin of the tibia. (B) Osteotomy was advanced with an osteotome to $5 \mathrm{~mm}$ medial to the lateral cortex. (C) Two osteotomes were placed in the osteotomy site to spread the osteotomy site without collapsing cutting surface and making intra-articular fracture and lateral hinge tears. (D) The medial tibia was fixed with a Puddu plate.

Table 2. Result of Autogenous Bone Graft Group and Allogenous Bone Graft Group

\begin{tabular}{|c|c|c|c|}
\hline Variable & Autogenous bone graft group $(\mathrm{n}=29)$ & Allogenous bone graft group $(n=23)$ & p-value \\
\hline \multicolumn{4}{|l|}{ Plate (mm) } \\
\hline 5 & 1 & 2 & \\
\hline 7.5 & 10 & 11 & \\
\hline 10 & 13 & 8 & \\
\hline 12.5 & 5 & 2 & \\
\hline Average & $9.4 \pm 1.97$ & $8.6 \pm 1.97$ & 0.147 \\
\hline Follow-up period (mo) & $28.2 \pm 6.22$ & $27.22 \pm 5.85$ & 0.529 \\
\hline Bone union period (wk) & $11.7 \pm 3.14$ & $12.1 \pm 2.90$ & 0.636 \\
\hline Reduction loss & $1(3.4)$ & $1(4.3)$ & \\
\hline Lateral cortex fracture & $4(13.8)$ & $3(13.0)$ & \\
\hline Meniscus operation & 23 & 22 & \\
\hline Microfracture \& OATs & 9 & 3 & \\
\hline Others & 9 & 5 & \\
\hline
\end{tabular}

Values are presented as mean \pm standard deviation or number (\%). p-value $<0.05$.

OAT: osteochondral autograft transplantation. 
of preoperatively planned lengths were obtained from the iliac crest on the contralateral side to facilitate the harvest and rapid rehabilitation and inserted anterior and posterior to the Puddu plate. The graft placed anterior to the plate was the shorter ${ }^{8)}$ than posterior and pushed to the medial end of the tibia using an impactor to prevent step-off, and the remaining space was filled with cancellous bone obtained from the iliac crest. In group II, freeze-dried allogenous cancellous bone chip (TranZgraft; Tissue Banks International, Baltimore, MD, USA) was inserted taking care to avoid destruction of the cancellous bone.

From the second postoperative day, range of motion exercises were started with a motion control brace worn in both groups. Although the range of flexion was gradually increased unless severe pain was caused, more than $90^{\circ}$ flexion was not permitted for the two postoperative weeks. In spite of the possibility of spontaneously healing after osteotomy ${ }^{9)}$, the combined posterior root tears of the medial meniscus were suture repaired to alleviate mechanical symptoms, and microfracture was performed for full thickness chondral defect as the subchondral bone was exposed (Table 2). Weight-bearing was not allowed for knees that underwent microfracture or meniscus suture repairs for six postoperative weeks, whereas partial weight-bearing was permitted for the remaining knees as long as it does not cause surgical site tenderness and pain during walking. Full weight-bearing was initiated if progressive partial weight-bearing did not cause pain at the osteotomy site and radiographic evidence of bone union was observed.

\section{Assessments}

The anatomical and mechanical femorotibial angles were mea-
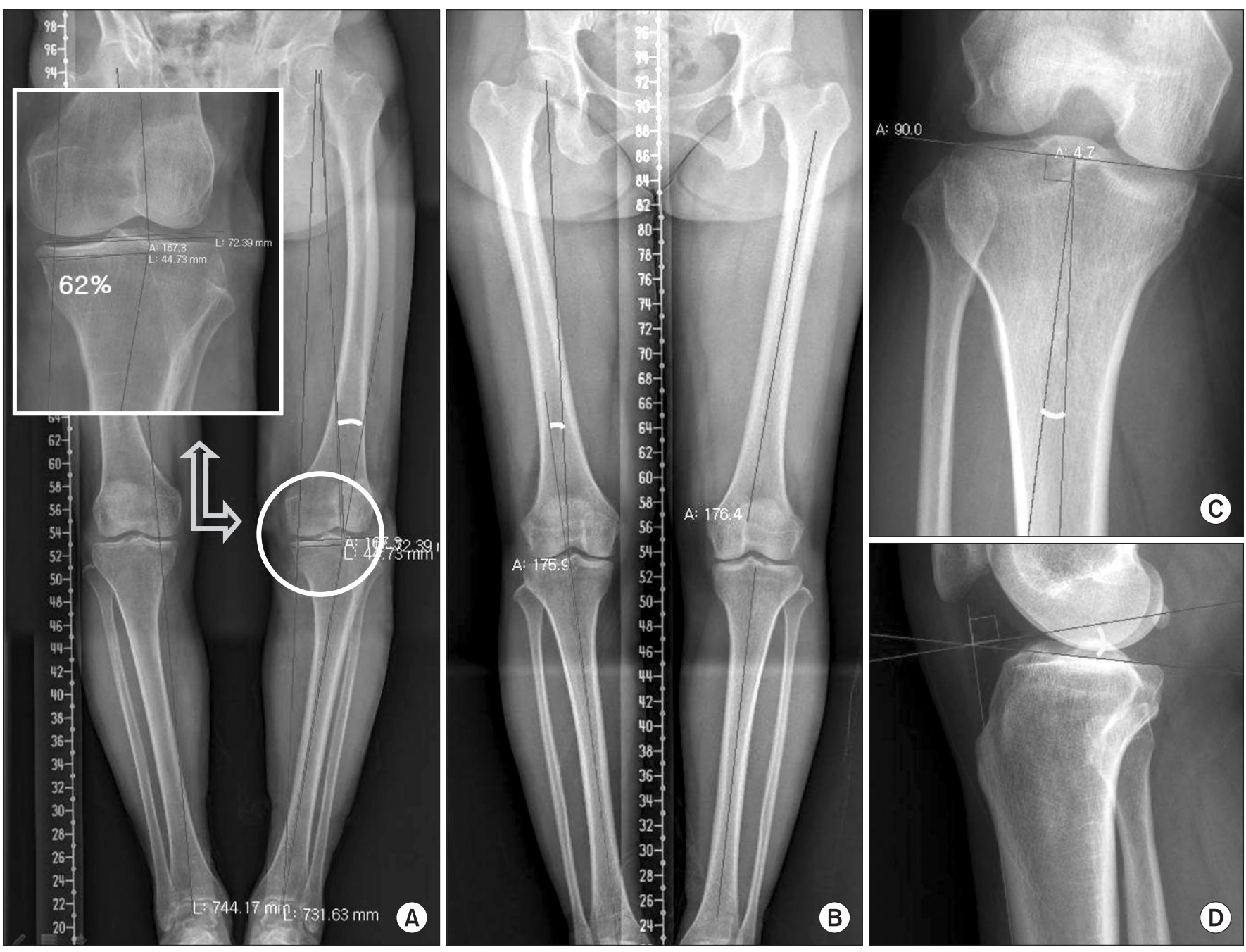

Fig. 2. Preoperative correction angle (A), mechanical femorotibial angle and anatomical femorotibial angle (B), modified tibial bone varus angle (C), and posterior tibial slope (D) were measured on radiographs. 
sured on weight-bearing whole-leg radiographs preoperatively, 6 weeks postoperatively, and at the last follow-up. The modified tibial bone varus angle and posterior tibial slope were assessed on the anteroposterior (AP) and lateral views. The tibial bone varus angle is generally measured as the angle between a line connecting the midpoint of the tibial plateau and that of the tibial epiphysis and a line parallel to the tibial shaft on the AP view. In this study, we defined the modified tibial bone varus angle as the angle created by a line perpendicular to a line tangent to the mediolateral articular surface of the tibial plateau and a line parallel to the tibial shaft on the AP radiograph. Posterior tibial slope was defined as the angle made by a line tangent to the tibial plateau and a line perpendicular to the anterior cortex of the tibia on the lateral radiograph (Fig. 2). All radiographic assessments were done twice each by two orthopedic surgeons with an interval of one week and the mean values were recorded.

On the clinical assessment, the visual analog scale (VAS) score and Knee Society score (KSS) ${ }^{10)}$ were evaluated immediately postoperatively, 2 weeks postoperatively, 6 weeks postoperatively, and at the last follow-up, and the occurrence of complications was investigated. Clinical bone union that was defined as no tenderness at the surgical site and pain during walking was considered as a prerequisite for partial weight-bearing, whereas radiological bone union defined as trabecular bone formation at the graft site was considered appropriate for full weight-bearing (Fig. 3). Loss of reduction was defined as displacement at the osteotomy site or $\geq 3^{\circ}$ decrease in the femorotibial angle on the weight-bearing whole-leg radiograph.

\section{Statistical Analysis}

A chi-square test was used to assess the anatomical and mechanical femorotibial angles, modified tibial bone varus angle, posterior tibial slope, bony union period, VAS score, and KSS. A $\mathrm{p}<0.05$ was considered statistically significant. The intraobserver and interobserver reliability for radiographic measurements was assessed using the Levene's test. All statistical analyses were performed using SPSS ver. 18.0 (SPSS Inc., Chicago, IL, USA).

\section{Results}

The mean metal plate size was not significantly different between group I and group II (9.4 mm vs. $8.6 \mathrm{~mm}$; $\mathrm{p}=0.147)$. The mean follow-up period was 28.2 months in group I and 27.7 months in group II. Radiographic evidence of bone union was observed in all cases (52 knees of 51 patients). The mean bone union period was 11.7 weeks in group I and 12.1 weeks in group
II, showing no significant difference ( $\mathrm{p}=0.636$ ) (Table 2).

There was no significant intergroup difference in the mean anatomical and mechanical femorotibial angles preoperatively, postoperatively and at the last follow-up ( $p>0.05$ ). The mean change in the anatomical femorotibial angle between the preoperative and postoperative period was $9.1^{\circ}$ in group I and $8.7^{\circ}$ in group II, showing no significant intergroup difference $(p=0.451)$. The mean decrease in the anatomical femorotibial angle between the last follow-up and immediate postoperative measurement was $1.2^{\circ}$ in group I and $1.8^{\circ}$ in group II, indicating no notable difference $(p=0.377)$. The mean change in the mechanical femorotibial angle between the preoperative and postoperative period was $9.6^{\circ}$ in group I and $9.2^{\circ}$ in group II, showing no significant intergroup difference $(p=0.713)$. The mean decrease in the mechanical femorotibial angle between the last follow-up and immediate postoperative measurement was $1.8^{\circ}$ in group I and $1.5^{\circ}$ in group II, exhibiting no remarkable difference $(\mathrm{p}=0.828)$. The mean modified tibial bone varus angle and posterior tibial slope were not significantly different between the groups preoperatively, postoperatively, and at the last follow-up (Table 3).

On the clinical assessment, the mean VAS score was significantly different immediately after surgery between group I and group II (5.7 vs. $4.5 ; \mathrm{p}=0.006$ ), but showed no significant difference thereafter. There was no significant difference in the mean KSS between group I and group II (92.1 vs. 94.3; p=0.419). Deep vein thrombosis developed in two knees in group I and no complication was observed in group II (Table 4).

Loss of reduction occurred in one knee (3.4\%) in group I and in one knee (4.3\%) in group II. Type I lateral cortical hinge tears where the fracture line reaches proximal to the tibiofibular joint ${ }^{11)}$ occurred in four knees (13.8\%) in group I and in three knees (13.0\%) in group II, which did not lead to loss of reduction (Table 2). There was no statistically significant intergroup difference in the intraobserver and interobserver reliability for radiographic measurements $(\mathrm{p}>0.05)$.

\section{Discussion}

High tibia osteotomy in knees with medial unicompartmental degenerative arthritis is effective for relieving symptoms by transferring the weight-bearing axis from the damaged medial compartment to the lateral side of the joint and delaying the need for a total knee arthroplasty (TKA) ${ }^{12)}$, allowing for later conversion to a TKA. Currently, open wedge techniques are more frequently employed than closed wedge techniques due to the following advantages: the risk of peroneal nerve injury is lower because the 
Table 3. The Result of Radiologic Assessment

\begin{tabular}{lccc}
\hline \multicolumn{1}{c}{ Variable } & Autogenous bone graft group & Allogenous bone graft group & p-value \\
\hline Anatomical FTA $\left(^{\circ}\right)$ & & & \\
Preoperative & $0.6 \pm 3.39$ & $1.1 \pm 3.19$ & 0.531 \\
Postoperative & $9.7 \pm 2.97$ & $9.8 \pm 3.38$ & 0.948 \\
Last follow-up & $8.5 \pm 3.18$ & $8.0 \pm 3.32$ & 0.612 \\
$\Delta$ Postoperative-preoperative & $9.1 \pm 2.91$ & $8.7 \pm 3.42$ & 0.451 \\
$\Delta$ Last follow-up-postoperative & $1.2 \pm 1.67$ & $1.8 \pm 1.53$ & 0.377 \\
Mechanical FTA $\left(^{\circ}\right)$ & & & 0.160 \\
Preoperative & $-7.3 \pm 2.88$ & $-5.6 \pm 3.08$ & 0.085 \\
Postoperative & $2.3 \pm 1.83$ & $3.6 \pm 2.49$ & 0.061 \\
Last follow-up & $0.5 \pm 1.50$ & $2.1 \pm 2.01$ & 0.713 \\
$\Delta$ Postoperative-preoperative & $9.6 \pm 2.61$ & $9.2 \pm 3.68$ & 0.828 \\
$\Delta$ Last follow-up-postoperative & $1.8 \pm 1.08$ & $1.5 \pm 1.82$ & \\
Modified tibia varus angle $\left({ }^{\circ}\right)$ & & & 0.202 \\
Preoperative & $-3.7 \pm 2.29$ & $-4.0 \pm 1.30$ & 0.425 \\
Postoperative & $1.8 \pm 2.78$ & $2.7 \pm 2.32$ & 0.128 \\
Last follow-up & $2.1 \pm 2.57$ & $3.0 \pm 2.40$ & 0.137 \\
Posterior slop of tibia $\left({ }^{\circ}\right)$ & & & $15.7 \pm 3.92$ \\
Preoperative & $14.2 \pm 4.42$ & $14.4 \pm 4.76$ & $15.9 \pm 5.78$ \\
Postoperative & $16.8 \pm 5.48$ & & \\
Last follow-up & $17.6 \pm 5.65$ & & \\
\hline
\end{tabular}

Values are presented as mean \pm standard deviation. p-value $<0.05$.

Negative (-) value means varus angle and positive (+) value means valgus angle.

FTA: femorotibial angle.

Table 4. The Result of Clinical Assessment

\begin{tabular}{lccc}
\hline & Autogenous bone graft group & Allogenous bone graft group & p-value \\
\hline Visual analog scale & $3.4 \pm 1.13$ & $2.7 \pm 1.00$ & 0.216 \\
Postoperative & $5.7 \pm 1.67$ & $4.5 \pm 1.14$ & 0.006 \\
Postoperative 2 wk & $3.2 \pm 1.73$ & $2.6 \pm 1.05$ & 0.144 \\
Postoperative 6 wk & $2.1 \pm 2.14$ & $1.8 \pm 1.25$ & 0.544 \\
Last follow-up & $2.5 \pm 1.78$ & $1.9 \pm 1.52$ & 0.204 \\
Korean Society score & $92.1 \pm 10.90$ & $94.3 \pm 6.78$ & 0.419 \\
Complication & 2 & 0 & 0 \\
\hline
\end{tabular}

Values are presented as mean \pm standard deviation . p-value $<0.05$.

proximal tibia and proximal tibiofibular joint remain unaffected; more accurate angular correction is attainable; and the techniques are easy to perform ${ }^{13,14)}$. On the other hand, the drawbacks include that bone grafting at the osteotomy site is required and the incidence of nonunion depends on the type of the graft material used ${ }^{1)}$. Although El-Assal et al. ${ }^{15)}$ reported satisfactory results of OWHTO up to $14 \mathrm{~mm}$ without bone grafting, the study did not include patients with obesity or poor bone quality, and bone grafting has been performed in most studies.

Autogenous grafts harvested from the iliac crest have been considered as the standard graft material in OWHTO, producing satisfactory outcomes ${ }^{16)}$. However, due to the prolonged surgical 

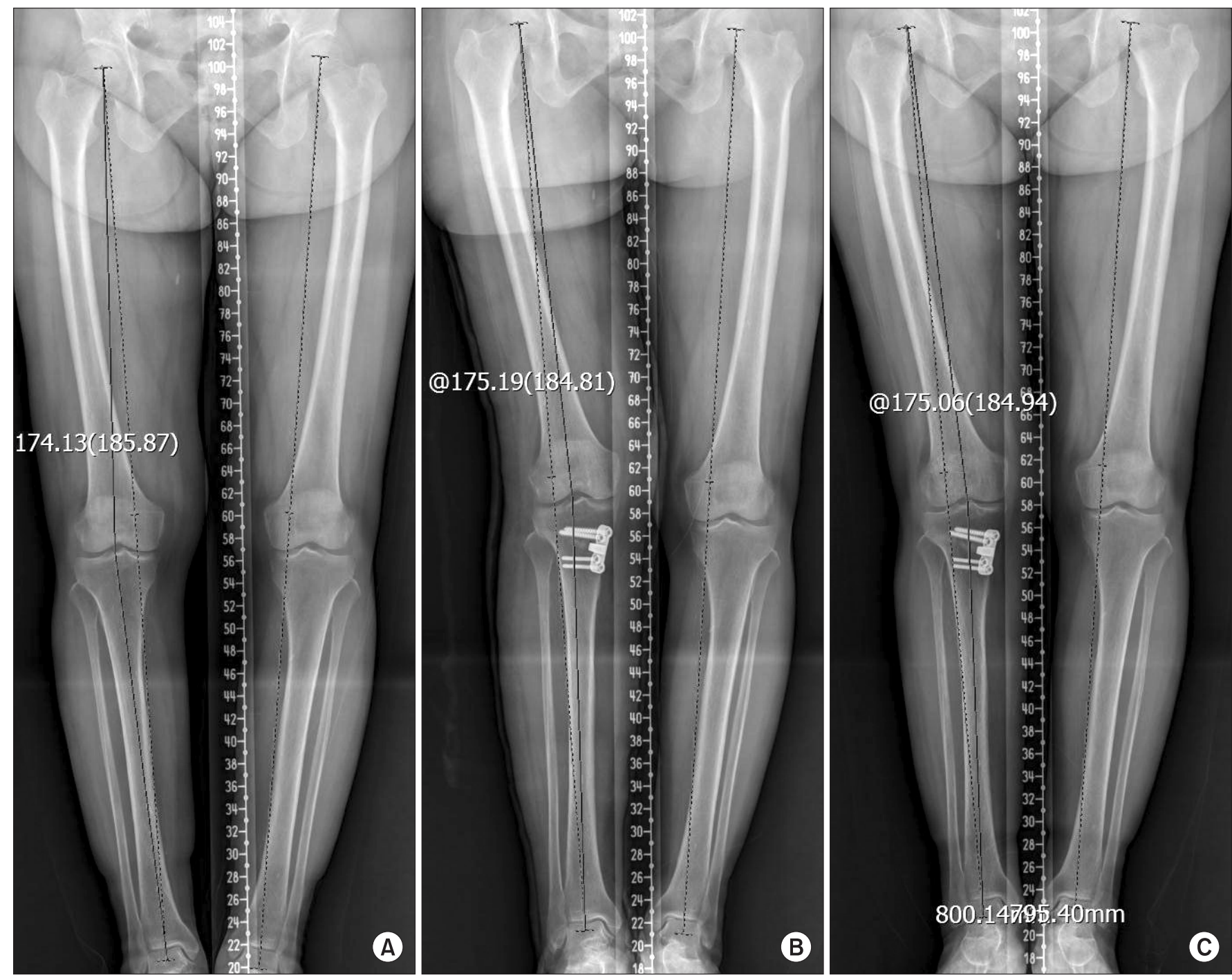

Fig. 3. (A) The preoperative standing whole leg anteroposterior (AP) radiograph obtained in a 67-year-old woman shows the mechanical femorotibial angle is $5.8^{\circ}$ varus. (B) The AP radiograph obtained 8 weeks after open wedge high tibial osteotomy using a 10.0-mm Puddu plate with allogenous bone chip (15 mL) shows the mechanical femorotibial angle is $4.8^{\circ}$ valgus. (C) The AP radiograph obtained 25 months postoperatively shows the mechanical femorotibial angle is $4.9^{\circ}$ valgus.

time for graft harvesting, donor site pain, and the risk of inflammation ${ }^{17-19)}$, allogenous bone grafts have been increasingly suggested as an alternative in domestic and foreign studies ${ }^{20-22)}$. Their advantages over autogenous bone grafts include no concern for donor site pain and theoretically unlimited supply, whereas the disadvantages are low bone formation rate, the risk of spreading disease, and high cost. On the other hand, there are commonly used graft substitutes, such as tricalcium phosphate $(\mathrm{TCP})^{2}$, bicalcium phosphate granule, acylic bone cement ${ }^{3)}$, and hydroxyapatite ${ }^{4)}$. Takeuchi et al. ${ }^{23)}$ compared stress assessed at five designated points between a group with a $7.5 \mathrm{~mm} \beta$-TCP wedge inserted into the osteotomy site and the other group without grafting: the stress on the medial plate and the lateral cortical hinge was reduced by $2 / 3$ in the former group. Thus, they advocated insertion of a graft into the osteotomy site for mechanical stability. Gouin et al. ${ }^{1)}$ compared the efficacy of a ceramic wedge (hyroxyapatitie $+\beta-$ TCP) and an autogenous graft in OWHTO: surgical site pain until the 6th postoperative month was greater in the ceramic wedge group; the incidence of loss of correction was higher in the ceramic group (6 of 22 knees) than in the autogenous graft group ( 1 of 18 knees); and the VAS score for assessing the postoperative iliac crest pain was decreased from 4.8 immediately postoperatively to 1.9 on the 5 th postoperative day. In our study, pain was evaluated by VAS regardless of the graft harvest site and osteotomy site. 


\section{Cho et al. Auto and Allogenous Cancellous Bone Graft in HTO}

The immediate postoperative VAS score was significantly higher in group I (5.7) than in group II $(4.5, \mathrm{p}=0.006)$. Pain was greater at the graft harvest site than the osteotomy site in most patients, but it was improved by the 2nd postoperative week, showing no statistically significant difference. In a study by Kuremsky et al. ${ }^{5}$, the failure rate of OWHTO using a Puddu plate was six times higher in the allogenous graft group than in the autogenous graft group ( $29.4 \%$ vs. $5.3 \%$ ), and lateral cortical breach and $\geq 11 \mathrm{~mm}$ large wedge size were attributed to the failure. In our study, the failure rate was relatively low and showed no significant difference between the allogenous graft group and the autogenous graft group ( $3.4 \%$ vs. $4.3 \%$ ), which could be attributed to the fact that the wedge size was $<12.5 \mathrm{~mm}$ in most of the patients and the incidence of lateral cortical breach was low in both autogenous graft group 13.8\% (4 cases) and allogenous graft group 13.0\% (3 cases) based on the study by Kuremsky et al. ${ }^{5)}$ (Table 2). In our opinion, the incidence of lateral cortical breach could have been affected by the surgical technique. Unlike other authors including Kuremsky et al. ${ }^{5)}$ who left $1 \mathrm{~cm}$ of the lateral cortex intact, we extended the osteotomy line to $5 \mathrm{~mm}$ medial to the lateral tibial cortex by gradually advancing an osteotome between two osteotomes (Fig. 1C). Accordingly, the incidence of lateral cortical hinge tears was relatively low, significant loss of correction was relatively rare because nondisplaced tears occurred without the ostoeotmy line extending to the intraarticular region or distal to the proximal tibiofibular joint, and there was no case of nonunion. Lee et al. ${ }^{21)}$ reported good short-term follow-up results of OWHTO using the TomoFix plate (High Medial Tibia, Synthes, Oberdorf, Switzerland) and an allogenous graft in knees that required $\geq 15^{\circ}$ of correction. Thus, it is our understanding that the Puddu plate is appropriate for small correction in high tibial osteotomy, whereas the use of the TomoFix plate should be taken into consideration for rigid fixation when the lateral cortex can be compromised for large correction. There was no significant difference in the mean bone union period between group I and group II (11.7 weeks vs.12.1 weeks).

The mean change between the 6 th weeks postoperative and last follow-up radiographic measurements of the anatomical femorotibial angle was $1.2^{\circ}$ in group I and $1.8^{\circ}$ in group II. The mean change in the mechanical feromotibial angle was $1.8^{\circ}$ varus in group I and $1.3^{\circ}$ varus in group II. These results showed that nearly neutral or slightly valgus mechanical femorotibial alignment was observed by 2 years after surgery in knees with excessive valgus correction regardless of the graft type.

In group I, $3.2^{\circ}$ of loss of reduction occurred in one knee. Although the patient complained of pain, bone union was observed and thus the implant was removed seven months after surgery. In group II, $3.4^{\circ}$ of loss of reduction was observed in one knee with minor pain, which has been followed up without additional treatment.

On the clinical assessment, the mean VAS score at the last follow-up was 2.4 in group I and 1.8 in group II, and the mean KSS at the last follow-up was 92.1 in group I and 94.2 in group II, showing no significant difference. In a study by Gouin et al. ${ }^{1}$, the calcium-phosphate graft and the autogenous graft exhibited similar clinical outcomes by two years after surgery.

Contrary to our hypothesis, allogenous grafts yielded comparable results to autogenous grafts in terms of the clinical and radiographic results and bone union period. In addition, the former caused less pain immediately after surgery.

The limitations of this study include the following: the study design was retrospective; patients' jobs or activity levels were not taken into consideration for grouping; the number of cases with autogenous grafts was higher in males than females because more male patients who are relatively less fearful of pain and wound chose to receive autogenous grafts; when there was gap between the size of Puddu plate with an interval of $2.5 \mathrm{~mm}$ and the actual correction angle with an interval of $1 \mathrm{~mm}$, the surgeon determined the plate size intraoperatively; there might have been errors in determining the bone union period based on the medical history of the patients with different follow-up periods; and the difference in the additional procedures for the combined injuries might have affected the clinical results.

\section{Conclusions}

Medial OWHTO using allogenous cancellous bone grafts for medial unicompartmental osteoarthritis of the knee can be considered as an alternative treatment method that provides equivalent radiographic and clinical results of medial OWHTO using autogenous bone grafts and results in less immediate postoperative pain.

\section{Conflict of Interest}

No potential conflict of interest relevant to this article was reported.

\section{Acknowledgments}

This study was supported by research funds from Chosun University Hospital 2010. 


\section{References}

1. Gouin F, Yaouanc F, Waast D, Melchior B, Delecrin J, Passuti N. Open wedge high tibial osteotomies: Calcium-phosphate ceramic spacer versus autologous bonegraft. Orthop Traumatol Surg Res. 2010;96:637-45.

2. Rejda BV, Peelen JG, de Groot K. Tri-calcium phosphate as a bone substitute. J Bioeng. 1977;1:93-7.

3. Hernigou P, Ma W. Open wedge tibial osteotomy with acrylic bone cement as bone substitute. Knee. 2001;8:103-10.

4. Koshino T, Murase T, Saito T. Medial opening-wedge high tibial osteotomy with use of porous hydroxyapatite to treat medial compartment osteoarthritis of the knee. J Bone Joint Surg Am. 2003;85:78-85.

5. Kuremsky MA, Schaller TM, Hall CC, Roehr BA, Masonis JL. Comparison of autograft vs allograft in opening-wedge high tibial osteotomy. J Arthroplasty. 2010;25:951-7.

6. Fujisawa Y, Masuhara K, Shiomi S. The effect of high tibial osteotomy on osteoarthritis of the knee: an arthroscopic study of 54 knee joints. Orthop Clin North Am. 1979;10:585608.

7. Asik M, Sen C, Kilic B, Goksan SB, Ciftci F, Taser OF. High tibial osteotomy with Puddu plate for the treatment of varus gonarthrosis. Knee Surg Sports Traumatol Arthrosc. 2006;14:948-54.

8. Chae DJ, Shetty GM, Lee DB, Choi HW, Han SB, Nha KW. Tibial slope and patellar height after opening wedge high tibia osteotomy using autologous tricortical iliac bone graft. Knee. 2008;15:128-33.

9. Nha KW, Lee YS, Hwang DH, Kwon JH, Chae DJ, Park YJ, Kim JI. Second-look arthroscopic findings after open-wedge high tibia osteotomy focusing on the posterior root tears of the medial meniscus. Arthroscopy. 2013;29:226-31.

10. Marti CB, Gautier E, Wachtl SW, Jakob RP. Accuracy of frontal and sagittal plane correction in open-wedge high tibial osteotomy. Arthroscopy. 2004;20:366-72.

11. Takeuchi R, Ishikawa H, Kumagai K, Yamaguchi Y, Chiba N, Akamatsu Y, Saito T. Fractures around the lateral cortical hinge after a medial opening-wedge high tibial osteotomy: a new classification of lateral hinge fracture. Arthroscopy. 2012;28:85-94.

12. Noda T, Yasuda S, Nagano K, Takahara Y, Namba Y, Inoue H. Clinico-radiological study of total knee arthroplasty after high tibial osteotomy. J Orthop Sci. 2000;5:25-36.

13. Brinkman JM, Lobenhoffer P, Agneskirchner JD, Staubli AE, Wymenga $A B$, van Heerwaarden RJ. Osteotomies around the knee: patient selection, stability of fixation and bone healing in high tibial osteotomies. J Bone Joint Surg Br. 2008;90:1548-57.

14. Naudie DD, Amendola A, Fowler PJ. Opening wedge high tibial osteotomy for symptomatic hyperextension-varus thrust. Am J Sports Med. 2004;32:60-70.

15. El-Assal MA, Khalifa YE, Abdel-Hamid MM, Said HG, Bakr HM. Opening-wedge high tibial osteotomy without bone graft. Knee Surg Sports Traumatol Arthrosc. 2010;18:961-6.

16. Hartford JM, Hester P, Watt PM, Hamilton D, Rohmiller M, Pienkowski D. Biomechanical superiority of plate fixation for proximal tibial osteotomy. Clin Orthop Relat Res. 2003;(412):125-30.

17. Chae DJ, Shetty GM, Wang KH, Montalban AS Jr, Kim JI, Nha KW. Early complications of medial opening wedge high tibial osteotomy using autologous tricortical iliac bone graft and T-plate fixation. Knee. 2011;18:278-84.

18. Goulet JA, Senunas LE, DeSilva GL, Greenfield ML. Autogenous iliac crest bone graft. Complications and functional assessment. Clin Orthop Relat Res. 1997;(339):76-81.

19. Silber JS, Anderson DG, Daffner SD, Brislin BT, Leland JM, Hilibrand AS, Vaccaro AR, Albert TJ. Donor site morbidity after anterior iliac crest bone harvest for single-level anterior cervical discectomy and fusion. Spine (Phila Pa 1976). 2003;28:134-9.

20. Ganji R, Omidvar M, Izadfar A, Alavinia SM. Opening wedge high tibial osteotomy using tibial wedge allograft: a case series study. Eur J Orthop Surg Traumatol. 2013;23:1114.

21. Lee JH, Cha YS, Lee JM, Seo ES. Early result of open wedge high tibial osteotomy using allogenous bone graft in patients with osteoarthritis of medial compartment of the knee. J Korean Musculoskelet Transplant Soc. 2011;11:43-9.

22. Santic V, Tudor A, Sestan B, Legovic D, Sirola L, Rakovac I. Bone allograft provides bone healing in the medial opening high tibial osteotomy. Int Orthop. 2010;34:225-9.

23. Takeuchi R, Bito H, Akamatsu Y, Shiraishi T, Morishita S, Koshino T, Saito T. In vitro stability of open wedge high tibial osteotomy with synthetic bone graft. Knee. 2010;17:21720. 